Ciudad y territorio 


\section{Regeneración urbana integrada: proyectos de acupuntura en Medellín}

Lic. María José Ramírez, Arquitecta

Invitada internacional

Universidad Católica del Norte, Chile

mjramirez@lovart.cl

Dra. Paula Kapstein, Arquitecta

Invitada Internacional

Universidad del Valparaíso, Chile.

paula.kapstein@uv.cl

Recibido: setiembre del 2015

Aceptado: mayo del 2016

Ciudad y territorio

Ensayo 


\title{
Resumen
}

En la última década, Medellín ha pasado de ser una ciudad de alta peligrosidad y con gran cantidad de problemas asociados a la vulnerabilidad social de sus barrios, a constituirse en un ejemplo de innovación en la gestión urbana, lo cual se traduce en la variedad de políticas y programas llevados a cabo con el objeto de regenerar sus barrios más pobres y de mejorar su accesibilidad, ya que la mayoría de estos barrios se sitúan en la periferia de la ciudad. Las actuaciones de acupuntura urbana, concebidas bajo la figura de planeamiento denominada Proyecto Urbano Integral (PUI), han sido capaces de reforzar elementos de resiliencia propios de los barrios, entre los cuales se cuenta el capital social. En este artículo se presenta la acupuntura urbana como un concepto referido a prácticas que son propias de la regeneración urbana integrada, considerando sus dos ejes: la intervención urbanística en la ciudad y la integración de elementos que permiten reforzar la cohesión social mejorando los procesos de identicación del ciudadano con su ciudad.

Palabras clave: acupuntura urbana; Medellín; proyectos urbanos integrales (PUI); regeneración.

\begin{abstract}
In the last decade Medellin has transformed itself from a dangerous city, suffering many problems associated with the social vulnerability of its neighborhoods, to a positive example urban innovation. This is a result of a wide range of programs specially created to regenerate the most vulnerable peripheral areas of the city, improving their accessibility. The urban acupuncture actions that were created under a new comprehensive urban planning tool (PUI), have been able to reinforce the elements of resilience and social capital already possessed by the neighborhoods. The article presents urban acupuncture as a concept congruent with practices carried out by an integral urban regeneration processes which focuses on two major subjects: the urban intervention in the city, and the integration of elements that make possible the reinforcement of social cohesion, while improving the citizen's identity within the city.
\end{abstract}

Keywords: urban acupuncture; Medellin; comprehensive urban planning tool (PUI); regeneration. 


\title{
Regeneración urbana integrada: proyectos de acupuntura en Medellín
}

\author{
Paula Kapstein ${ }^{1}$ \\ María José Ramírez ${ }^{2}$
}

\section{Introducción y objetivos}

Se busca, principalmente, definir el concepto de acupuntura dentro del marco de la regeneración urbana integrada ${ }^{3}$. Para ello, se consideran los siguientes objetivos:

- Reflexionar sobreel concepto de regeneración urbana integraday sus realizaciones, entre ellas el Proyecto Urbano Integral(PUI);

- $\quad$ Estudiar cómo la acupuntura urbana puede lograr una recuperación del entorno a nivel socio espacial en Medellín, referenciando este análisis con casos donde esta recuperación genera equilibrio de usos y funciones en el barrio.

En este artículo, primero, se verifica cómo la acupuntura urbana se inscribe en el marco más general de la regeneración para, a continuación, resaltar los aspectos cualitativos de esta práctica, lo que se ha denominado acupuntura de percepción (Ramírez, 2014), y que junto a los componentes de intervención espacial configuran el contexto de estrategias de la acupuntura.

\section{Sobre la gestión urbana en Medellín}

La ciudad de Medellín, situada en el Valle de Aburrá, Colombia, se compone de dieciséis comunas. Cada una de ellas, a su vez, se divide en barrios y en áreas institucionales. La

\footnotetext{
$1 \quad$ Paula Kapstein López, Arquitecta (Universidad de Valparaíso) y Doctora en Urbanismo por la Universidad Politécnica de Madrid, actualmente trabaja como docente e investigadora en la Facultad de Arquitectura de la Universidad de Valparaíso, desarrollando el proyecto Metodología para elaboración de Plan Estratégico de Regeneración Integral para el Gran Valparaíso. Realizó estadía posdoctoral en la Facultad de Arquitectura, Urbanismo y Artes de la Universidad Nacional de Ingeniería de Lima entre octubre de 2011 y junio de 2013 . Dicha estadía le permitió desarrollar la investigación Las Periferias Interiores de Lima: origen, estructura y dinámicas. Más tarde, entre los años 2013 y 2015, trabajó como profesora en la Escuela de Arquitectura de la Universidad Católica del Norte.

2 María José Ramírez Ljubetic, Licenciada en Arquitectura (Universidad Católica del Norte), actualmente cursando el último semestre de la carrera. Realizó su Seminario Acupuntura Urbana. Estrategias de equilibrio territorial en Antofagasta, bajo la tutoría de la Dra. Arquitecto Paula Kapstein López, el año 2014, tomando como ejemplo el rotundo cambio a nivel urbano que ha tenido la ciudad de Medellín los últimos años gracias al Urbanismo participativo. Desde aquel trabajo de investigación la alumna ha seguido la misma línea en su proyecto de título, ubicado en la ciudad de Antofagasta, Chile.

3 El seminario de investigación Acupuntura urbana. Estrategias de equilibrio territorial, de María José Ramírez (2014), abocado a estudiar las actuaciones de acupuntura urbana llevadas a cabo en Medellín en los últimos años para aplicarlas luego al diagnóstico de Antofagasta, constituye la base para el análisis que se hace aquí sobre la gestión urbana en Medellín. Este trabajo fue posible gracias al viaje de estudios a Medellín del taller integrado 89 de la carrera de Arquitectura de la UCN, realizado en abril de 2014 .
} 
ciudad tiene un total de 249 barrios oficiales y veinte áreas institucionales, las cuales se sitúan a ambos márgenes del cauce del río Medellín, ya que la ciudad se encuentra divida por el curso de este río.

En las décadas de los ochenta y noventa, Medellín fue escenario de guerras entre bandas de narcotraficantes, paramilitares, guerrilleros y sicarios, y cuna del cártel de droga más temido de América; sin embargo, esta ciudad es hoy un ejemplo de urbanismo contemporáneo gracias a un modelo centrado en la educación, el civismo y la convivencia.

Uno de los logros de la gestión referida a las infraestructuras, es el sistema de transporte, el cual está dirigido a mejorar la accesibilidad de los ciudadanos, atendiendo específicamente a la movilidad urbana de las personas que viven en las zonas más alejadas.

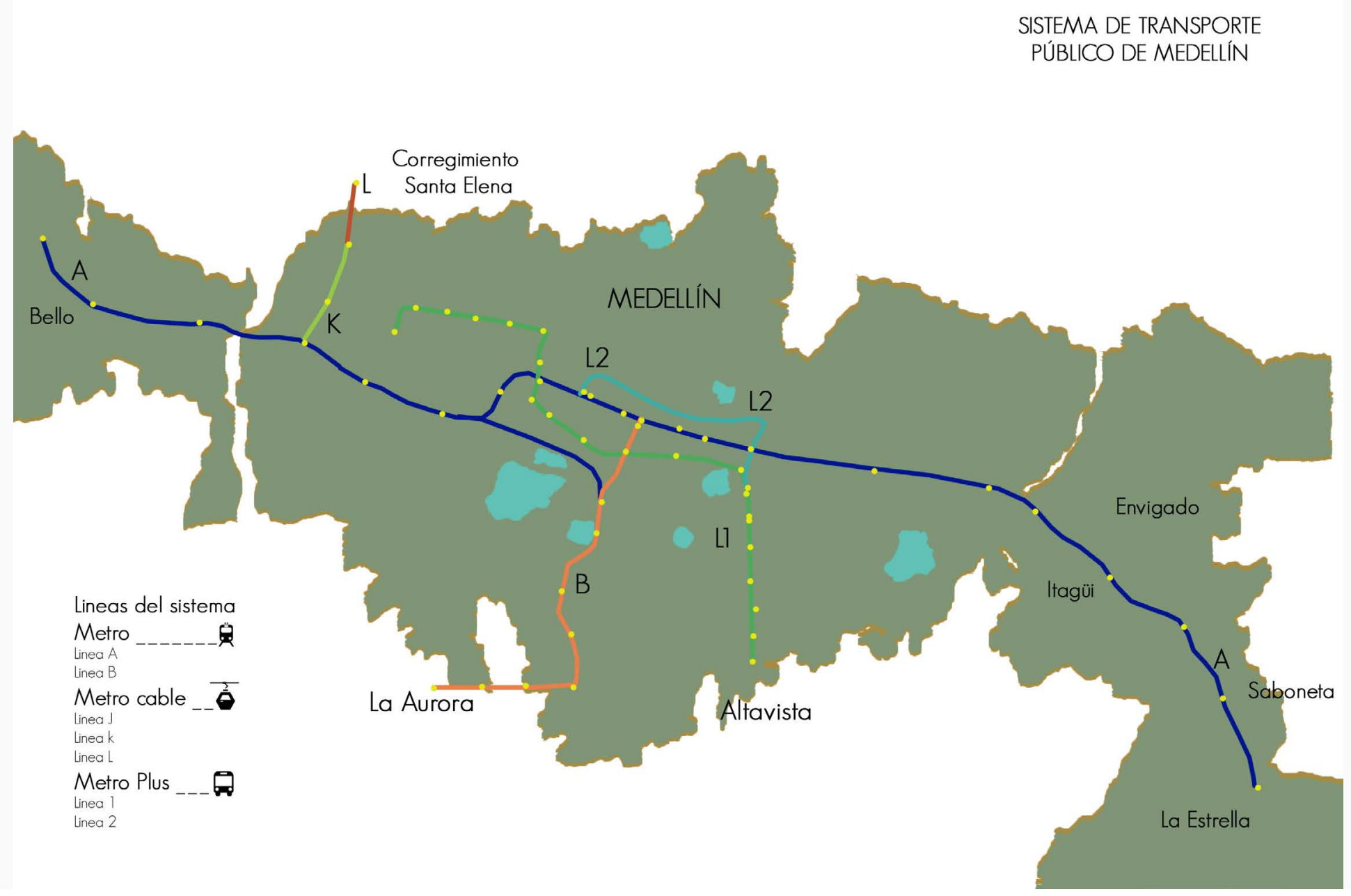

Figura 1. Mapa de la ciudad de Medellín y de sus líneas de transporte (metro, metroplús y metro cable). Elaborado por María José Ramírez de acuerdo a plano del Metro de Medellín y del Foro Urbano WUF 7, Medellín, 2014.

En el implante de estas soluciones de transporte y en la transformación que ha tenido la ciudad en los últimos años es destacable la alta participación ciudadana y la gestión de entidades como la Empresa de Desarrollo Urbano (EDU)4.

4 La EDU es una entidad de carácter público-privado relacionada con la Alcaldía de Medellín. Se encarga de la gestión de proyectos de regeneración urbana en Medellín. 


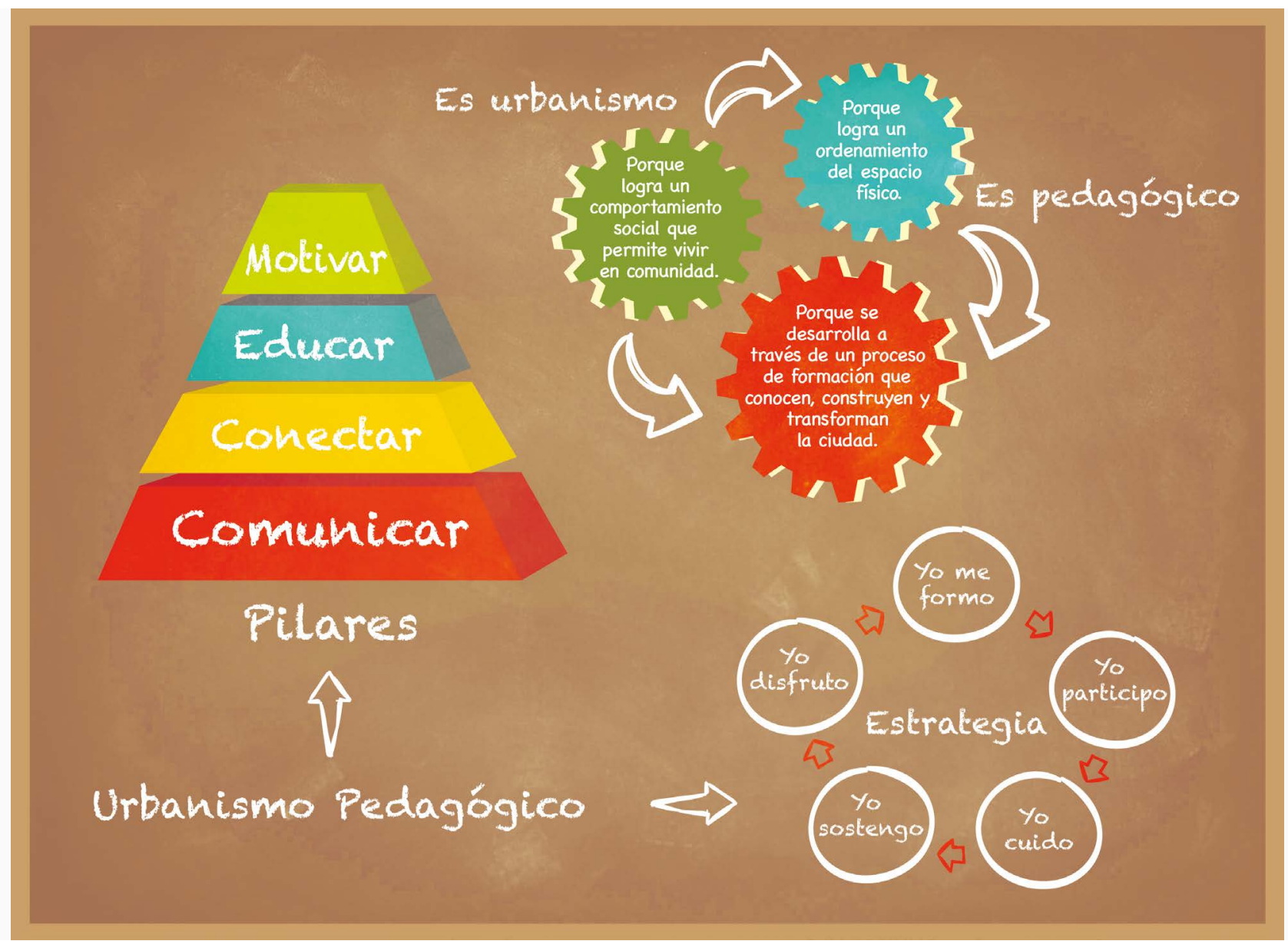

Figura 2. Esquema explicativo del concepto de Urbanismo Social. Elaborado por María José Ramírez de acuerdo a esquema de la EDU, 2014.

Uno de los aspectos más relevantes de la gestión urbana en Medellín es la incorporación del concepto de Urbanismo Social, el cual se aplica en la puesta en marcha de diferentes programas educativos y culturales, en los que no se concibe la propuesta de cambios en la ciudad sin la adecuada preparación de los ciudadanos para contribuir en este proceso (figura 2). Las referencias de este planteamiento son, por una parte, lo enunciado en la Declaratoria de las ciudades educadoras (Génova, 2004); y, por otro lado, los conceptos de pedagogía social (Morón, 2005), pedagogía urbana (Cuesta, 2010) y ética urbana (Uribe, Molina y Correa, 2011). Estas propuestas se basan en la convicción de que hoy las ciudades disponen de incontables posibilidades educadoras. De una forma u otra, las ciudades presentan, desde las propias características que las definen, elementos importantes para una formación integral de sus ciudadanos. En este sentido, Medellín, por su propia complejidad, se ha constituido en los últimos años en un agente educativo permanente y plural.

\section{Regeneración urbana: el Proyecto Urbano Integral PUI}

La práctica de la regeneración urbana se define como el conjunto de acciones integradas que, aplicadas a una zona urbana en crisis, pretenden frenar las dinámicas y los factores de declive, reactivando la capacidad local para afrontar los cambios urbanos, económicos y sociales (Hernández Aja et al., 2000). Esta definición nos interesa 
porque hace mención a la cuestión de la integración de estrategias; si se considera que un proyecto de regeneración no sólo atiende problemas urbanos sino también problemáticas ambientales y sociales, entonces su esencia está en la transversalidad e integración de directrices y planes, lo cual se define por los propios objetivos del proyecto de regeneración urbana. Por otro lado, es incuestionable el papel que la regeneración juega en la recuperación económica de las ciudades. Según Castrillo, Matesanz, Sevilla y Sánchez (2014), las instituciones plantean en la actualidad los procesos de regeneración como un elemento clave en la construcción de un modelo basado en la sostenibilidad y la integración de los aspectos sociales, económicos y ambientales. En cuanto a los aspectos ambientales, es importante también considerar el aporte de autores como Haase, Frantzeskaki y Elmqvist (2014) que analizan la brecha existente entre la urbanización y el abastecimiento de servicios ecosistémicos en regiones urbanas, donde la gobernanza y el planeamiento focalizado en los beneficios que los ecosistemas generan resultan vitales en un escenario de demanda de recursos y energía cada vez mayores. En este contexto, la puesta en valor de estos servicios en la gestión y planificación de metrópolis y de regiones urbanas puede incidir en un notable aumento en la calidad de vida de las personas desde la sostenibilidad y el rescate de los valores paisajísticos y los recursos naturales de una zona. Asimismo, se considera que la implantación de servicios ecosistémicos en el marco de la gestión urbana de escala metropolitana e intercomunal permitiría desarrollar planes de regeneración urbana integrada, capaces de generar estrategias que aúnen innovación tecnológica, urbana, social y ambiental.

Enmarcándose en el concepto de regeneración urbana, en Medellín se ha desarrollado, en los últimos años, la figura del Proyecto Urbano Integral(PUI) que corresponde a un instrumento multidimensional de prospección social, diálogo y planificación destinado a la intervención urbana, principalmente en áreas socialmente vulnerables. Por ello, este instrumento abarca las dimensiones de lo social, lo institucional y lo físico, con el fin de resolver problemáticas específicas en una zona definida. La metodología utilizada está diseñada para que la comunidad tenga un rol preponderante dentro del proceso de regeneración, lo que legitima cada una de las intervenciones desarrolladas y permite que estos proyectos sean detonantes de una convivencia ciudadana mejor. La estrategia principal del Proyecto Urbano Integral es entender las potencialidades del espacio público para reactivar nuevos centros urbanos en zonas vulnerables de la ciudad. La clave de sus intervenciones está dada por la provisión de equipamientos públicos ${ }^{5}$, mejorando la accesibilidad del barrio al mismo tiempo que se introducen 
cambios que le otorgan cualidades al espacio de la calle, humanizándolo: otorgando al peatón los espacios que necesita para caminar con tranquilidad o permanecer, disfrutando de su ciudad.

El Proyecto Urbano Integral(PUI) se considera un instrumento de regeneración urbana integrada por su capacidad de intervenir en diferentes escalas dentro de la ciudad (vecindarios, barrios y distritos), por la variedad de actuaciones que caben en dicho proyecto y porque activa la cohesión social. Un buen ejemplo de esto lo constituyen los Parques Biblioteca, los cuales son centros culturales emplazados en algunos de los barrios más vulnerables de la ciudad de Medellín (barrios con altos índice de pobreza y de falta de accesibilidad). Fueron creados con el fin de generar un fuerte desarrollo social capaz de fomentar el encuentro ciudadano y las actividades educativas y lúdicas.

\section{Acupuntura urbana}

Jaime Lerner acuñó el concepto de acupuntura urbana. "Es importante entender que una ciudad se puede cambiar inmediatamente a través de la acupuntura urbana; se puede crear una nueva energía en poco tiempo con algunas acciones focales... No es necesario gastar muchos recursos" (Lerner, 2006: minuto 15).

El arquitecto e investigador finlandés, Marco Casagrande, fue también de los primeros autores que colaboraron en la definición del concepto de acupuntura urbana, defendiendo un enfoque más local y socialmente participativo en la renovación urbana, justo cuando comenzaba el último periodo de crisis económica europea, a mediados de la década del 2000.

La premisa central de las actuaciones de acupuntura urbana es que se interviene para revitalizar, haciendo que todas las partes del barrio interactúen de un modo sinérgico. Por otro lado, la idea de que las intervenciones de acupuntura urbana logran mejorar la participación de la colectividad, tiene que ver con lo que planteaban Jacobs, Cairns y Strebel (2007, p.610):

Si los habitantes de las zonas se apropian de estas plazas barriales, seguramente además de generar sentimientos de pertenencia y de dignidad, se producirán también sinergias que mejoren la seguridad, lográndose integración social a partir del encuentro con una persona diferente. 
Generalmente, la intervención del proyecto de acupuntura urbana apunta a resolver problemas en la escala del barrio, es decir, actúa en localizaciones puntuales que pueden mejorar un entorno mayor; su estrategia de actuación busca entender primero las necesidades de los vecinos, para luego analizar cuáles pueden ser las potencialidades del espacio público, con el fin de regenerar zonas en declive. Lo anterior se relaciona con un tipo de planificación de escala reducida, la micro-planeación, que se compone de intervenciones pequeñas y localizadas. Esta, según Goethert, Hamdi et al.(1992), consiste en un proceso de desarrollo urbano basado en la comunidad, con programas para el mejoramiento comunitario que deben ser preparados e implantados en la localidad de manera cooperativa y rápida. Se trata de un proceso dimensionado a la escala debida para incentivar la capacidad administrativa local y la toma de decisiones capaces de estructurar el trabajo del proyecto en sí.

Se consideran tres componentes de la acupuntura urbana, referidos a los ámbitos social, urbano y físico. Asimismo, se concibe vulnerabilidad como "aquella situación crítica dada por problemas en los ámbitos social, físico y urbanístico de una ciudad, que se caracteriza por su complejidad, dada en una superposición de hechos relacionados: desigualdad social, degradación del medio físico y fragmentación del espacio urbano" (Kapstein, 2010, p.27). Deacuerdoa esta definición se puede relacionar conceptualmente la acupuntura con la vulnerabilidad urbana:

Social: Desdeeste punto devista la vulnerabilidad se da por una situación de desigualdad, la que genera falta de oportunidades e impide que las personas que la sufren tengan medios para superarla, o incluso que puedan acceder a la información necesaria para ello. Toda intervención a modo de acupuntura urbana debe comenzar con un estudio sobre la identidad social del barrio donde se emplazará. De este modo, el proyecto de acupuntura debe nacer de las necesidades de los habitantes; por esto es imprescindible realizar un trabajo participativo con la comunidad de vecinos antes, durante y después de la intervención.

Urbano: La vulnerabilidad urbana se expresa cuando el espacio de la ciudad se degrada funcional o socialmente. Esto, principalmente, es producto de procesos económicos que propician la especulación con el suelo y la vivienda. La acupuntura urbana puede ofrecer respuestas innovadoras de regeneración e integración de estas zonas segregadas.

Físico: Este componente se considera en cuanto a los aspectos de configuración geomorfológica del territorio y respecto al estado medioambiental de la ciudad y la existencia de servicios ecosistémicos o su carencia. Las características topográficas del 
emplazamiento del lugar, sumadas a las propiedades del suelo y a las características medioambientales del sitio, generan un cuadro de exposición a riesgos de toda índole, todo lo cual determina la vulnerabilidad física y medioambiental del lugar.

Las estrategias de acupuntura urbana buscan reducir la vulnerabilidad dada por estos tres componentes, integrando los sectores segregados al resto de la ciudad, aumentando su accesibilidad e, incluso, interviniendo en la propia configuración física del territorio cuando haga falta para prevenir problemas que se pueden presentar en un asentamiento bajo amenazas naturales: riesgos de inundación, desmoronamiento de terrenos, sismos, etc.

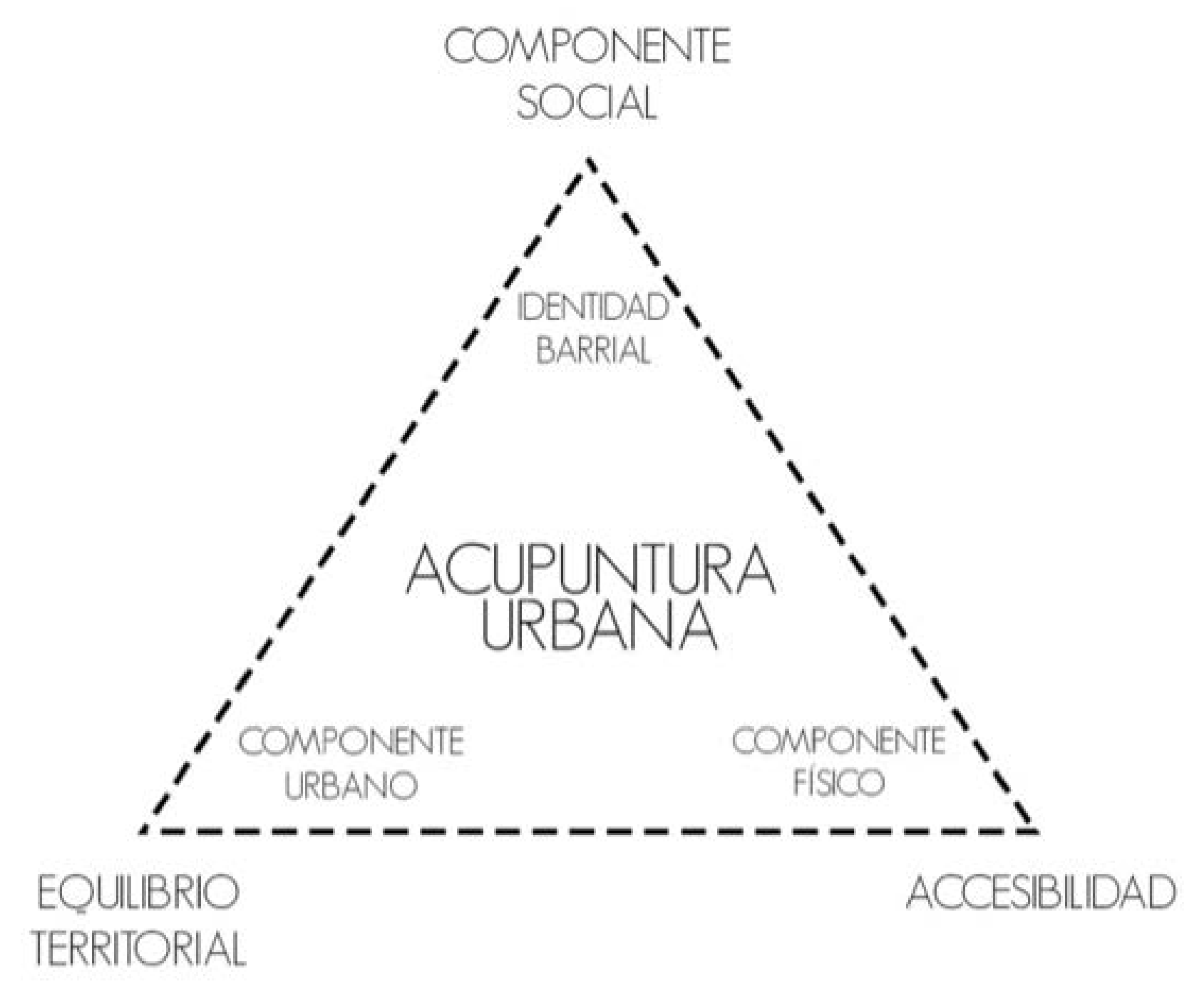

Figura 3. Componentes de la acupuntura urbana. Fuente: Ramírez, 2014

La integración de aspectos referidos a cada uno de los componentes en las estrategias que sustentan la práctica de la acupuntura permite que esta sea capaz de aunar una serie de valores con el objeto de lograr ciudades más sostenibles y menos fragmentadas, mejorando la calidad de vida y la integración social (figura 3). Estos son también los objetivos que persigue el Proyecto Urbano Integral, concretando generalmente las estrategias de la acupuntura; sin embargo, el PUI actúa en una escala distrital o comunal (ver figura 4). Hace falta que este instrumento incorpore en su gestión la micro-planeación ligada al concepto de acupuntura. La micro-planeación reúne actuaciones que por su pequeña escala de intervención pueden responder directamente 
a las necesidades reales de los vecinos; en este sentido, el PUI debería considerar esta escala en sus estrategias, ofreciendo alternativas a la autogestión del espacio público, de modo que el ciudadano pueda ser el protagonista en la regeneración de los espacios de su entorno directo: barrio, vecindario y acceso a su vivienda.

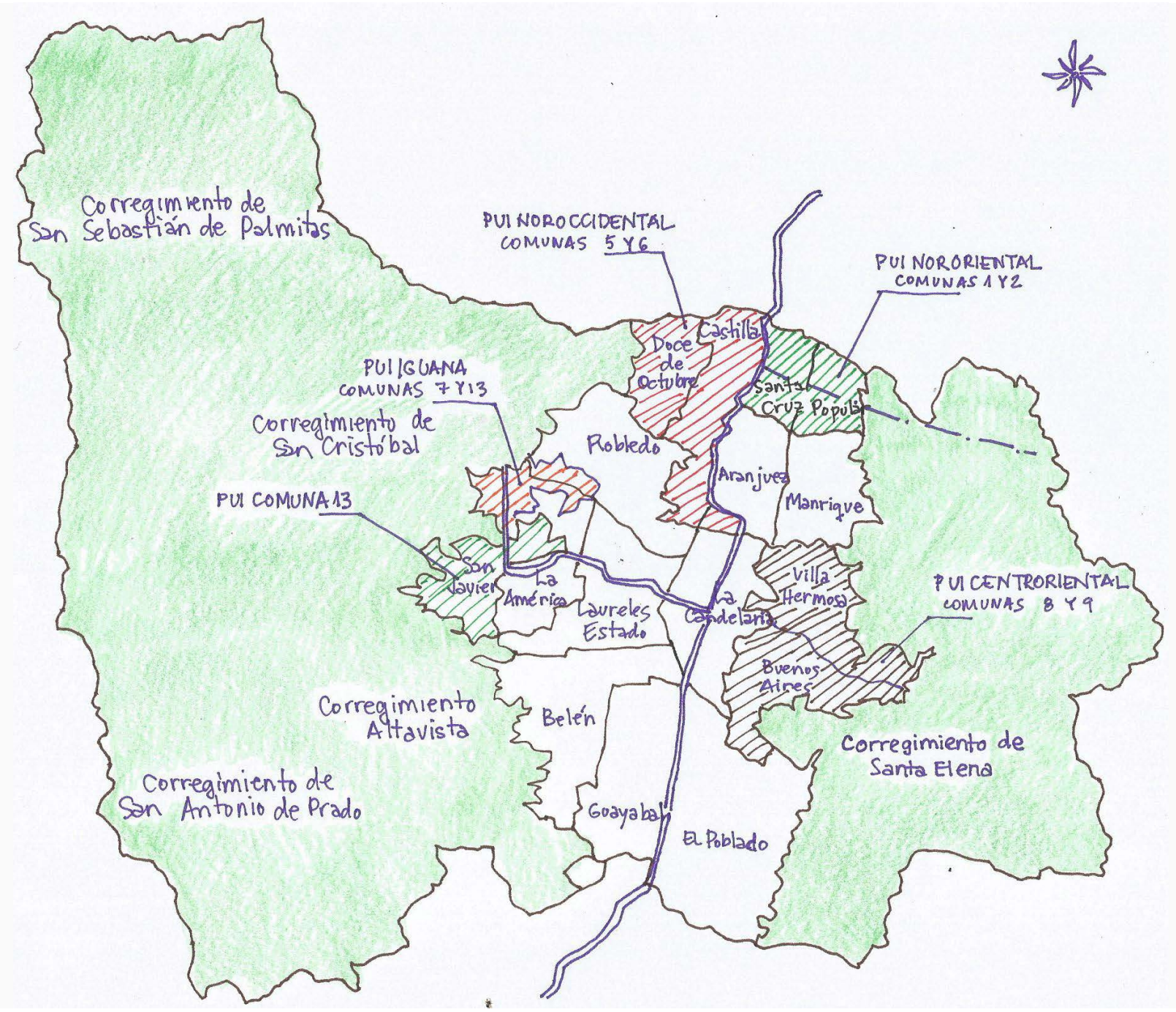

Figura 4. Ubicación de los proyectos urbanos integrales en Medellín. Elaborado por Paula Kapstein de acuerdo a plano de la EDU, 2015

\section{Tipos de acupuntura urbana}

De acuerdo a lo observado en la ciudad de Medellín, las actuaciones de acupuntura urbana pueden ser de dos tipos (Ramírez, 2014): de intervención, cuando se lleva a cabo una operación urbana capaz de revitalizar un barrio, creando nuevos espacios y puntos de encuentro y mejorando la accesibilidad; y de percepción, cuando se realizan intervenciones que no responden necesariamente a estrategias urbanas pero que permiten dotar de identidad local al sector, facilitándole al habitante una mejor lectura de su ciudad. La acupuntura de percepción recoge elementos propios de la memoria colectiva y la historia de un barrio. Generalmente, ambos tipos de actuaciones se complementan, como se da en el caso del Parque Explora (ver foto figura 5).

Los proyectos urbanos integrales, según Ramírez (2014), se pueden clasificar de acuerdo a los tipos de acupuntura urbana definidos. Así, el análisis de casos de acupuntura 
observados en la ciudad de Medellín permite valorar variables de intervención o de percepción. A continuación, se consideran tres casos donde están presentes las variables de accesibilidad, la transformación del espacio público y la presencia del agua en los parques. Estas son algunas de las variables que componen tanto la acupuntura de intervención como la de percepción. Según sea el peso de estas variables en la actuación es posible definir de qué tipo es cada acupuntura.

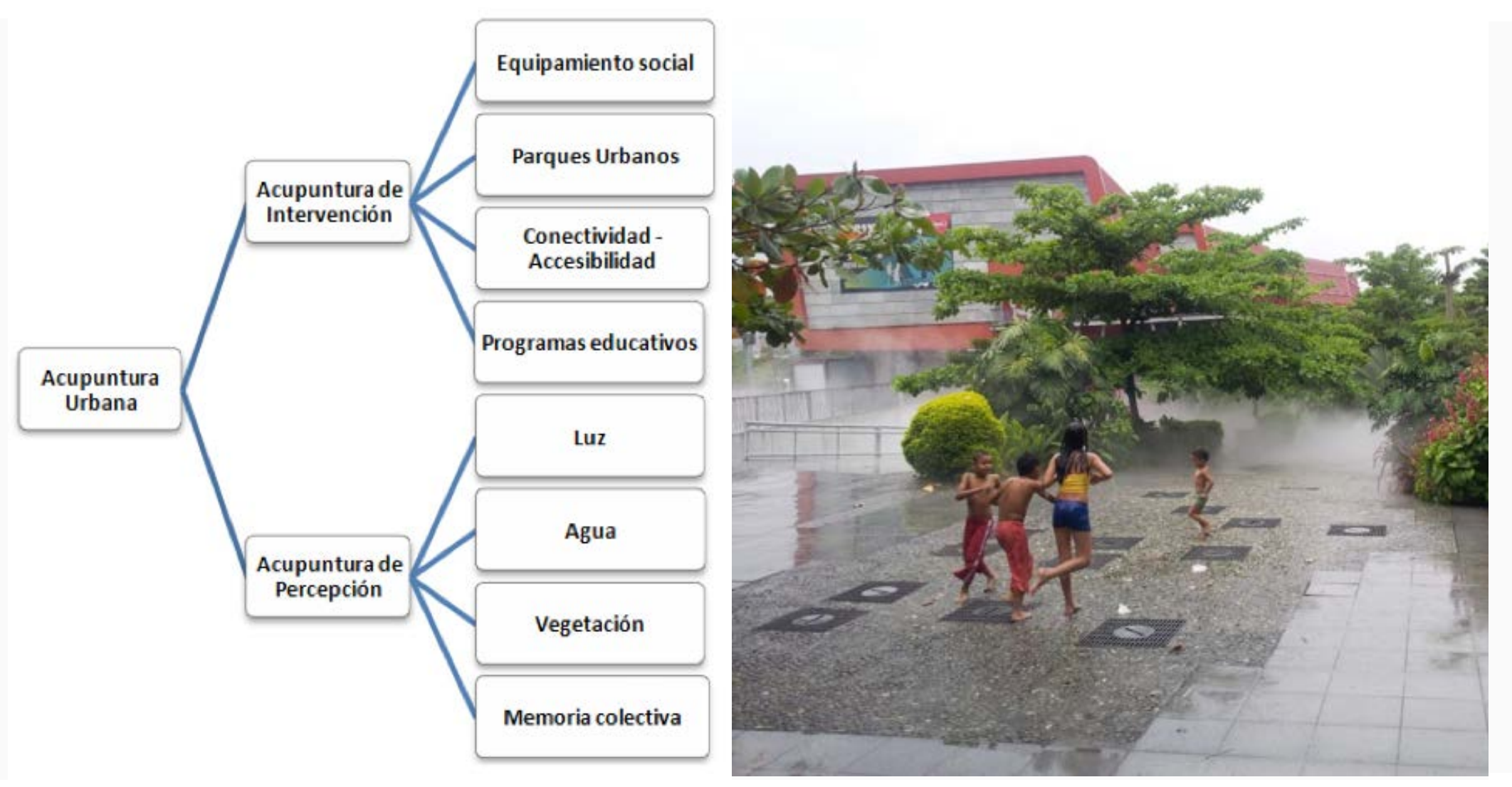

Figura 5. Esquema de tipos de acupuntura y sus componentes. Fuente: M.J. Ramírez, 2014. Imagen de niños jugando en una fuente de agua en el Parque Explora, ejemplo de acupuntura urbana integral (intervención + percepción). Foto: P. Kapstein, abril 2014.

\section{Comuna 13: mejora de la accesibilidad}

La movilidad urbana se entiende como factor de distribución y aprovechamiento del territorio, fomentando de manera equitativa los potenciales de la ciudad. Según Herce (2009, p.12) "reconocer que la movilidad urbana es un derecho ha sido la gran conquista social de los últimos años; y entender que movilidad y transporte no significan lo mismo es una consecuencia de esa conquista". En Medellín, aunque las redes de movilidad están pensadas para lograr la integración de todo su territorio, aún hay zonas periféricas que no cuentan con una buena accesibilidad y ello se traduce en marginación social. Sin embargo, se ha avanzado en los últimos años en este sentido, y un ejemplo de esto es la intervención en la Comuna 13, en el barrio de San Javier. El proyecto es utilizado por más de doce mil personas al día, contando a los habitantes del área de influencia de este sistema de movilidad y a los vecinos del barrio Independencias I. El origen de esta comuna se relaciona con las invasiones por parte de familias desplazadas por 
la violencia rural y se desarrolló con la construcción informal de asentamientos en las empinadas laderas de este sector. La intervención, principalmente, consistió en mejorar la accesibilidad de las zonas más altas del sector, incorporando tramos de escaleras cubiertas y algunos tramos de escaleras mecánicas descubiertas, además de concebirse la adecuación del espacio público circundante junto a un programa de recuperación de las fachadas y techumbres de las viviendas incluidas en el proyecto (figura 6). También se instalaron juegos infantiles en los espacios adyacentes a las escaleras.

Esta actuación es obra de la Alcaldía de Medellín y fue ejecutada por la Empresa de Desarrollo Urbano bajo la figura de Proyecto Urbano Integral(PUI).

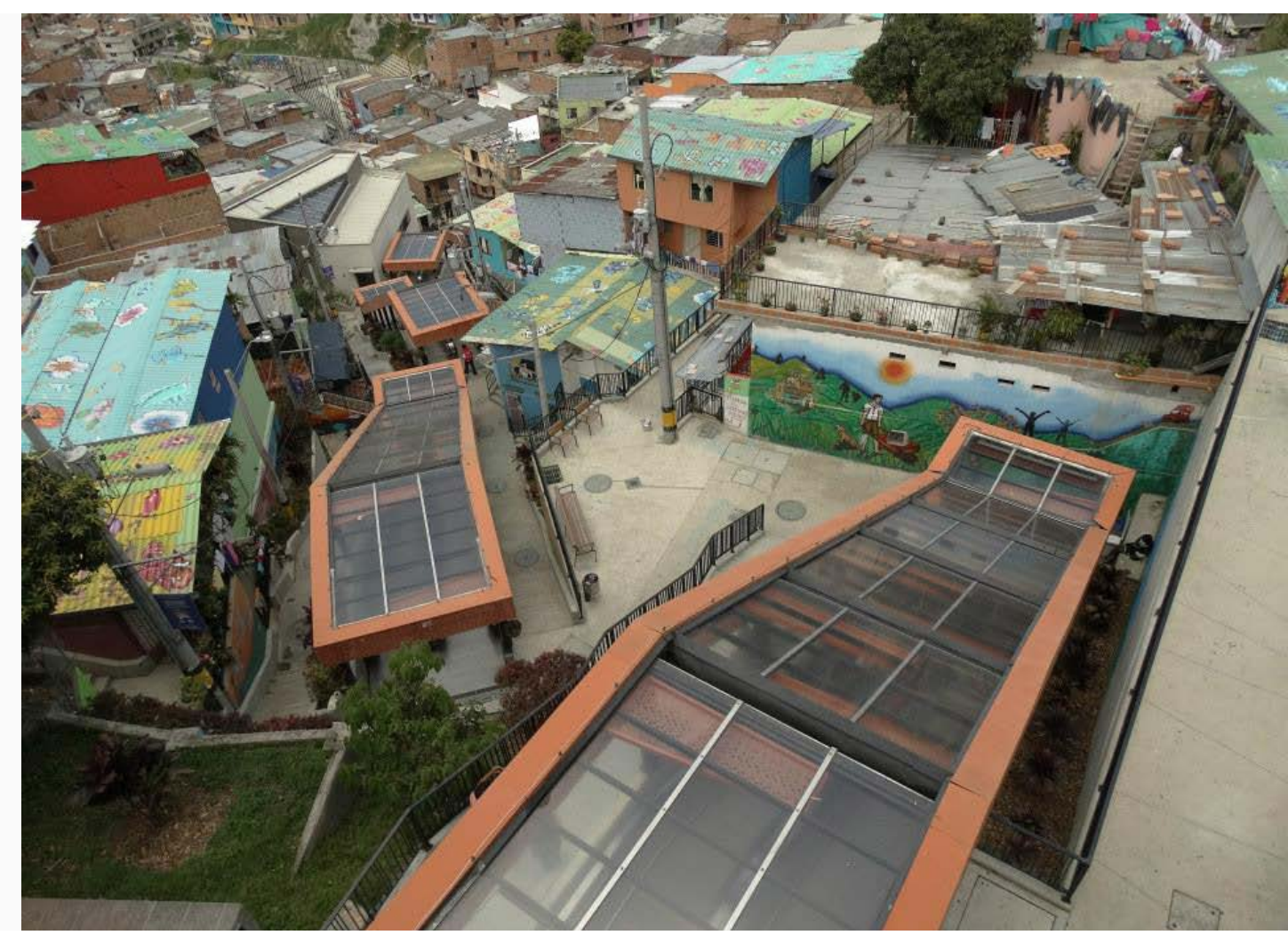

Figura 6. Escaleras mecánicas de la comuna 13 y adecuación del espacio público. Fuente: Nicole Boettcher, 2014.

Este caso se considera notable no solo por el esquema de movilidad propuesto que cumple un doble objetivo: mejorar la accesibilidad y potenciar la cohesión social del barrio, sino porque permite fomentar la implantación de medios de transporte sustentables de acuerdo a las necesidades de los vecinos de un determinado sector. Se trata claramente de un caso de acupuntura urbana de intervención.

\section{La transformación sustentable del espacio público: equilibrio entre intervención y percepción}

El espacio público, entendido como elemento estructurante de la ciudad, puede ser capaz de atender la diversidad de demandas de los ciudadanos referidas a la movilidad y a la permanencia en las calles desde una perspectiva socio espacial, donde la calidad del 
espacio urbano contribuye a que tengan una existencia digna, equitativa y sustentable. Además, cuando el espacio público es de calidad, permite el desarrollo de una amplia gama de actividades y que estas sean de larga duración (Gehl, 2008).

La presencia de los parques biblioteca y de parques y espacios rehabilitados en barrios socialmente vulnerables permite a los vecinos de más edad recordar historias sobre el origen de su barrio y a los jóvenes les lleva a conocer y probablemente también a identificarse con estos relatos, recomponiéndose así la identidad de su sector y su cohesión social a través del fortalecimiento de la memoria colectiva.

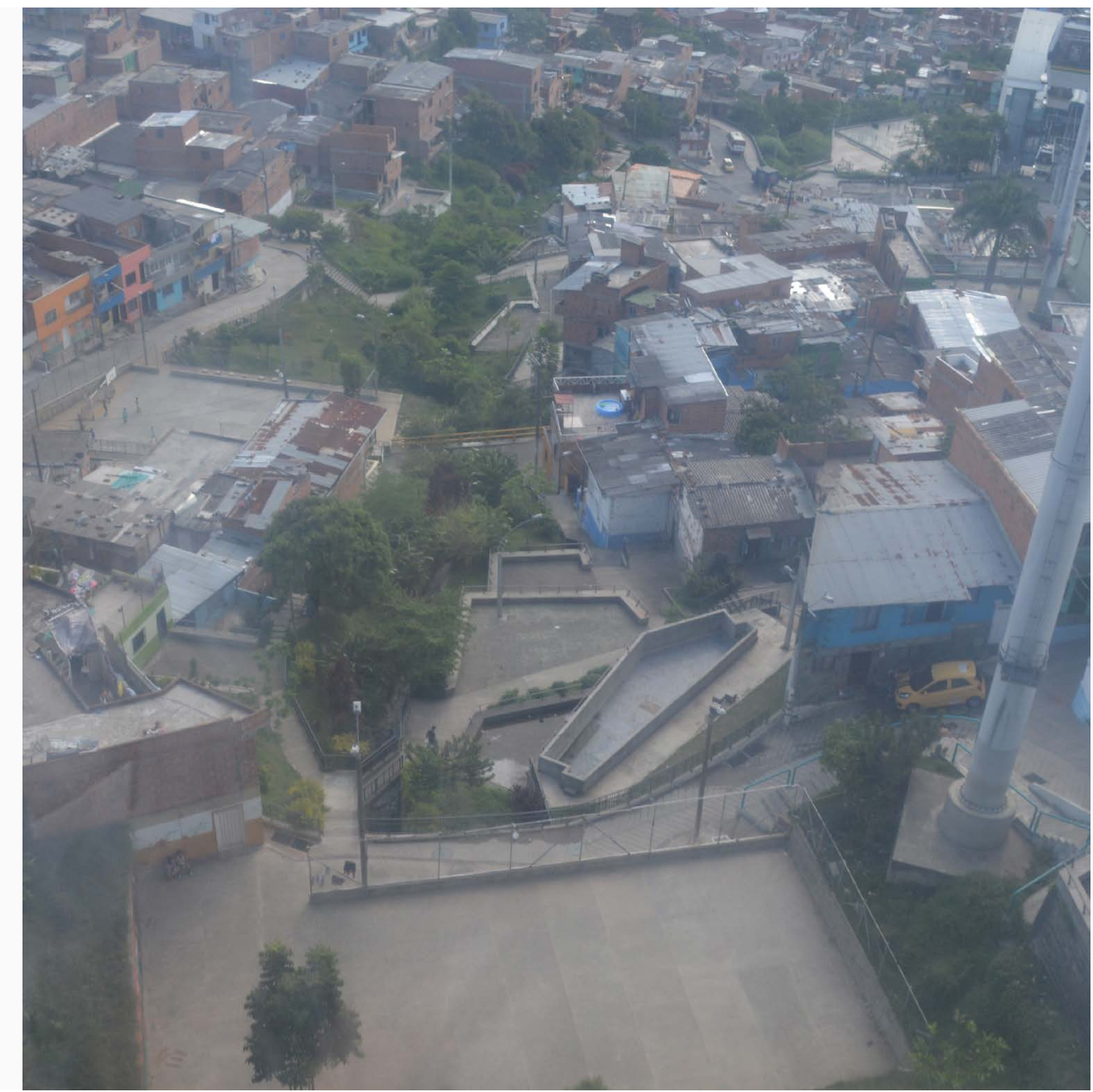

Figura 7. Parque lineal quebrada la Herrera. Imagen del estado actual de las piscinas. Fuente: Lautaro Ojeda, 2015.

Los parques urbanos, generalmente se componen de elementos propios de la acupuntura de intervención, pero también presentan rasgos de la acupuntura de percepción (con la incorporación de agua, vegetación y espacios lúdicos), se trata de casos donde ambos aspectos se suelen dar equilibrados. Sin embargo, en el caso del parque lineal quebrada la Herrera es importante observar que después de un primer impacto social y urbano, las piscinas han dejado de ser utilizadas porque presentaban algunos problemas constructivos y por el alto costo de su mantenimiento. Finalmente, la revitalización que busca la acupuntura en esta intervención no ha sido conseguida del todo. 


\section{Parques urbanos: el agua en la acupuntura de percepción}

El agua es uno de los elementos que componen la memoria ambiental de los habitantes de Medellín. En los parques Pies Descalzos y Explora, y en la Biblioteca Belén (figuras 8, 9 y 10), este elemento le otorga nuevas posibilidades y significados al lugar de encuentro, las cuales van más allá de los usos tradicionales. Estas posibilidades están determinadas por tres ideas clave: juego, educación y cultura, consolidándose el planteamiento de que el espacio público también puede educar a los ciudadanos cuando está diseñado para responder a sus necesidades de un modo amable e integral (Bustamente, 2015). También es importante considerar la incidencia que los parques de este tipo tienen en la recomposición de la memoria colectiva y en el fortalecimiento de los procesos de identificación del ciudadano con su ciudad. La identidad y el sentimiento de pertenencia a un lugar tienen que ver, en definitiva, con el hecho de que nuestros recuerdos van asociados a la ciudad que nos acoge y a nuestros barrios y, dentro de ellos, a lugares especiales que se constituyen como hitos en nuestra memoria. La acupuntura de percepción puede ser capaz de recoger los lugares que se han transformado en hitos de la memoria de un grupo de vecinos o ciudadanos y ponerlos en valor.

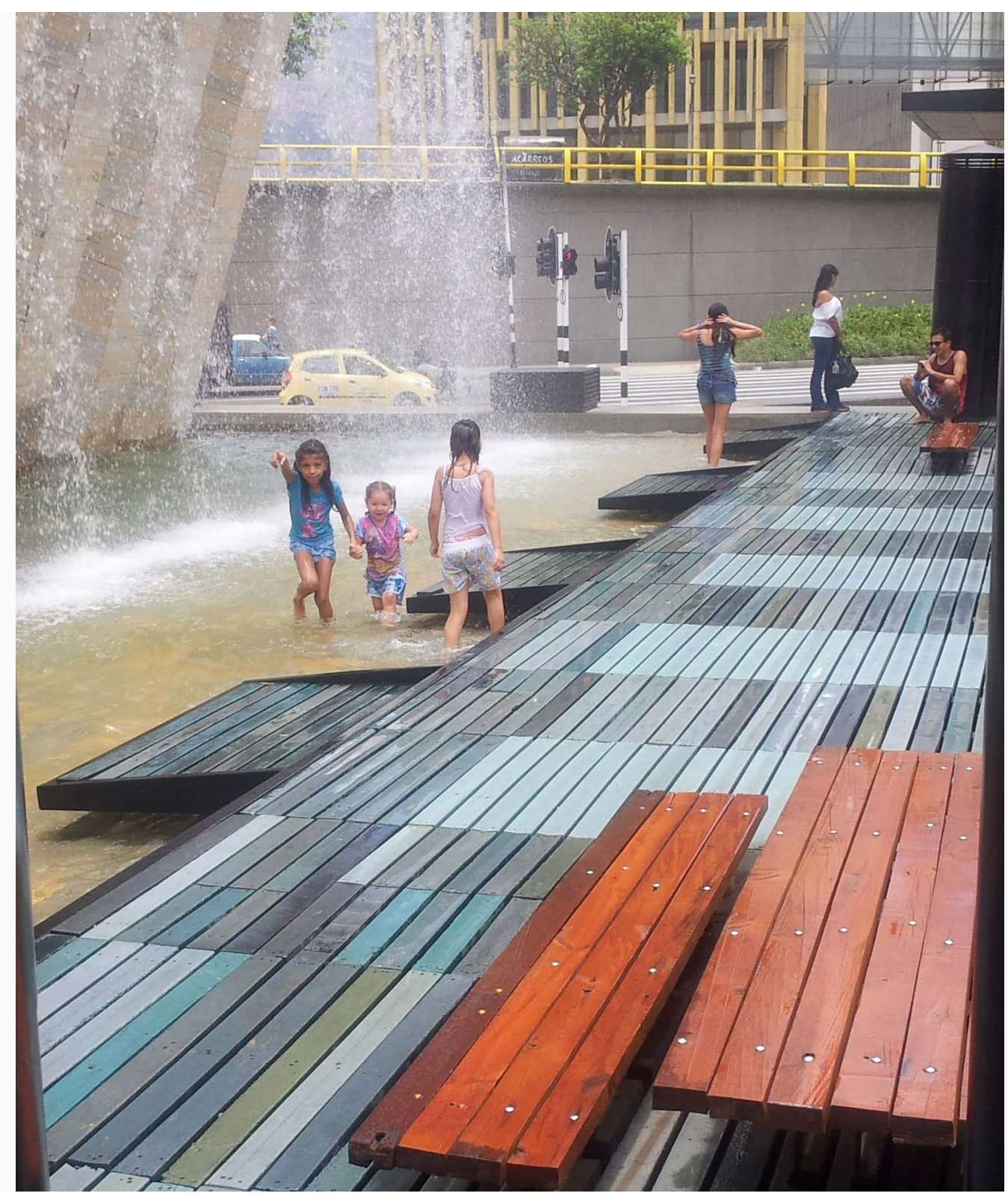

Figura 8. Fotografía del Parque Pies Descalzos. Fuente: Miguel Ángel Gálvez, 2014. 


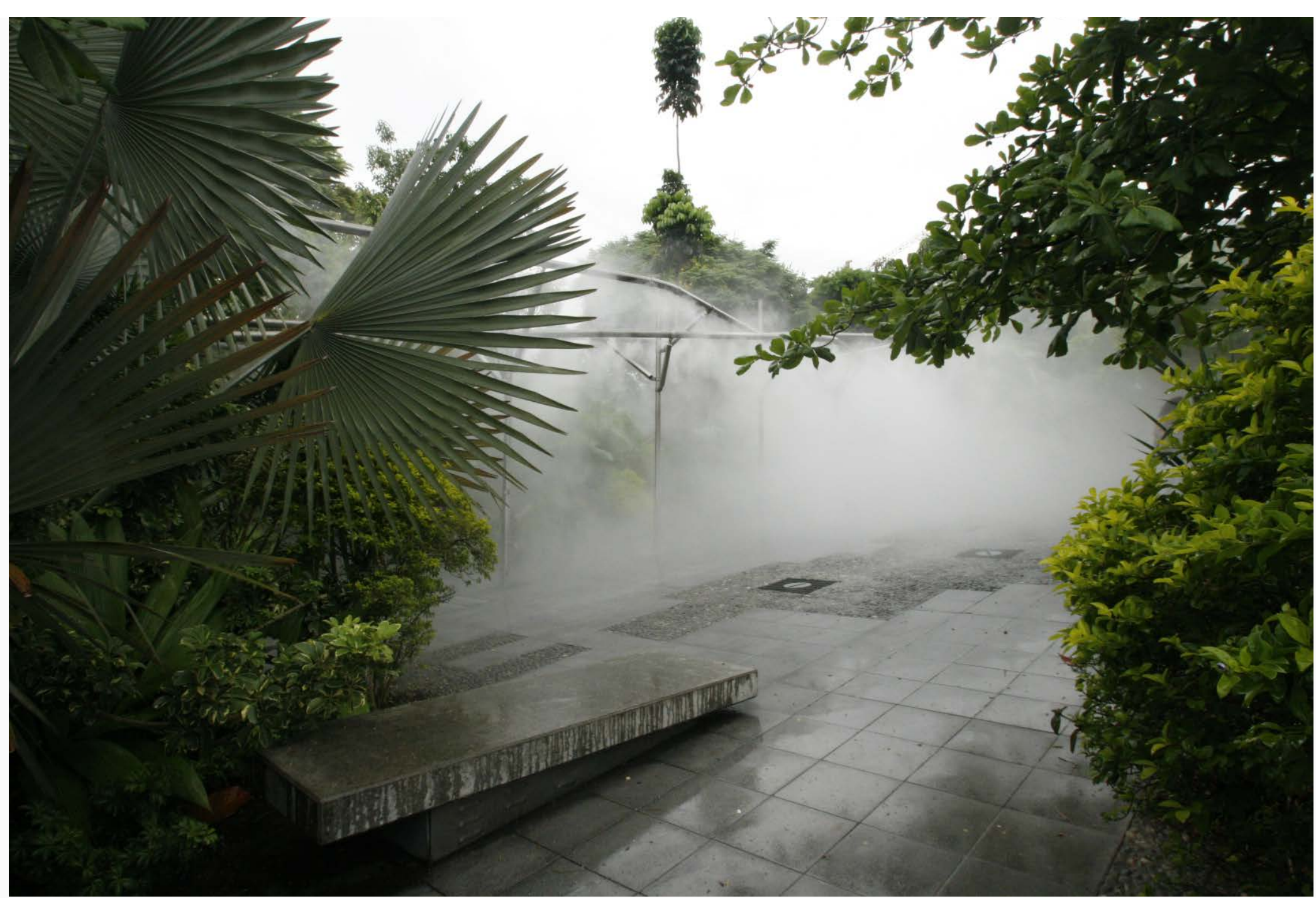

Figura 9. Parque Explora. Autor: Massimo Palme, 2014.

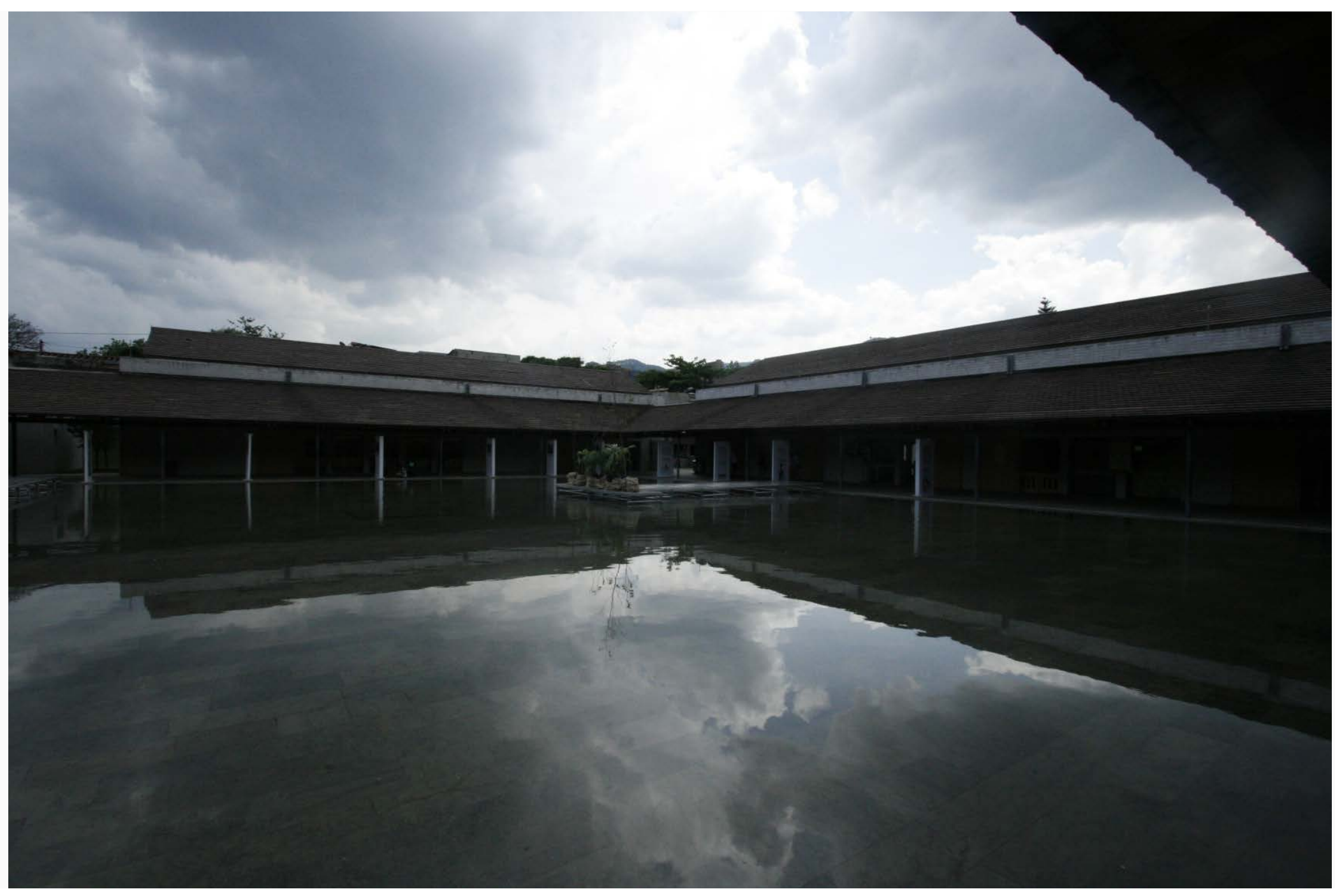

Figura 10. Fotografía del patio de agua de la Biblioteca Belén. Fuente: Massimo Palme, 2014 


\section{Consideraciones: propósitos de la acupuntura urbana}

Se han expuesto los componentes de la acupuntura urbana y sus características más importantes, definiendo dos tipos. Se resalta lo social de entre los componentes enunciados, ya que éste se refiere al desarrollo de la participación que se necesita para llevar a cabo la regeneración de un sector. En este sentido, se considera que la acupuntura tiene los siguientes propósitos integrales:

- $\quad$ El desarrollo urbano sostenible, que a su vez se basa en una equidad social, económica y física;

- El desarrollo de las necesidades humanas, en donde la acupuntura urbana puede actuar como satisfactor de las necesidades básicas, las que según Max-Neef (1998) son: subsistencia, protección, afecto, entendimiento, participación, ocio, creación, identidad y libertad;

- $\quad$ La mejora general en la calidad de vida de los ciudadanos.

\section{Conclusiones}

El caso de Medellín puede contribuir a la reflexión que debe acompañar la práctica de la regeneración urbana de las ciudades en Latinoamérica. Dicha práctica aún es reciente y tiene como antecedente lo que viene dándose desde hace tres décadas en las ciudades europeas. Citando a Mongil (2010, p.148):

La progresiva profundización de los procesos de segregación socio-espacial en las ciudades europeas ha supuesto que, a lo largo de los últimos años, se hayan ido consolidando políticas urbanas que, asumiendo las tradicionales intervenciones de renovación, rehabilitación y mejora urbana, han añadido la consideración de una dimensión socioeconómica a fin de no sólo mejorar las condiciones físicas de estos barrios sino también de frenar o, al menos, de mitigar los procesos que contribuyen a reforzar los problemas sociales, económicos, ambientales y urbanísticos.

De aquí, es importante observar que la regeneración urbana integrada debe ir acompañada de cambios en las políticas urbanas, que permitan la incorporación de leyes que la promuevan y de subvenciones estatales que la apoyen.

Se reconoce que todo proceso de mejoras de un barrio vulnerable resulta ser complejo y lento, porque intervienen múltiples factores dados por las redes sociales y humanas del lugar, los cuales no son abordables desde los instrumentos de planificación que conocemos. Se requiere de instrumentos aún más globales y cualitativos, que partan y 
terminen en la gente que va a recibir los cambios, ya que el urbanismo no puede limitar su acción a la mera provisión de espacios que cuantitativamente atiendan necesidades por demandas insatisfechas.

El urbanismo y la planificación urbana deberían fundar su acción en una relación paritaria entre los técnicos, los ciudadanos y la administración de la ciudad, de tal forma que todos ellos sean conscientes del significado, alcance y beneficio de las propuestas urbanas y arquitectónicas que se proponen y se llevan a cabo entre todos. Esto sólo se puede conseguir cuando los proyectos de regeneración urbana se acompañan de programas de educación cívica sostenidos en el tiempo, tal como se está haciendo en Medellín.

En cuanto al Proyecto Urbano Integral, tal y como se está abordando en Colombia, lo ideal sería extender dicha noción para incluir en el planeamiento un amplio tipo de proyectos complementarios al anterior, de diversa dimensión y complejidad. En este sentido, la acupuntura como práctica de la micro-planeación debería estar vinculada a planes o en proyectos de cierta jerarquía o envergadura dentro de la ciudad.

\section{Referentes}

Bustamente, S. (2015). Charla impartida en el taller de Planificación Urbana Integral, durante visita de equipo de urbanistas de URBAM, realizada en octubre a la Facultad de Arquitectura de la Universidad de Valparaíso de Chile, en el marco del proyecto Geropolis.

Castrillo, M., Matesanz, Á., Sánchez, D. y Sevilla, Á. (2014). ¿Regeneración urbana? Deconstrucción y reconstrucción de un concepto incuestionado. PAPELES de Relaciones ecosociales y cambio global, núm. 126, pp. 129 -139, FUHEM, Madrid.

Cuesta, O. (2010). Pedagogía urbana, convivencia ciudadana y aprendizaje por reglas. Revista Educación y Desarrollo Social, vol. 4, núm. 2, pp. 176-188, Julio - Diciembre, Bogotá. Consultado el 15 de abril de 2015. Disponible en http://www.umng.edu.co/ documents/63968/80125/art_13.pdf

Declaratoria de las ciudades educadoras, (2004). Consultada el 14 de mayo de 2015. Disponible en http://www.bcn.cat/edcities/aice/estatiques/espanyol/sec_charter. html 
Empresa de Desarrollo Urbano de Medellín y Banco Interamericano de Desarrollo, (2014). Equidad territorial en Medellín. Navarrete, J., Bernal, M. y Donovan, M. (eds.), Medellín.

Gehl, J. (2008). La humanización del espacio urbano. La vida social entre los edificios. Ed. Reverte, Barcelona.

Goethert, R., Hamdi, N., Gray, S., Slettebak, A., Wischmeier, W. H., Paz, J., ... у Soeteman, F. J. (1992). La microplanificación; un proceso de programación y desarrollo con base en la comunidad (No. GTZ-872). Banco Mundial, Washington, DC (EUA).

González Escobar, L. F., (2007). Medellín, los orígenes y la transición a la modernidad: crecimiento y modelos urbanos 1775-1932 (No. 27). Universidad Nacional de Colombia, Sede Medellín, Escuela del Hábitat-CEHAP.

Haase, D., Frantzeskaki, N. y Elmqvist, T. (2014). Ecosystem Services in Urban Landscapes: Practical Applications and Governance Implications. The Royal Swedish Academy of Sciences.

Herce, M. (2009). Sobre la movilidad en la ciudad: propuestas para recuperar un derecho ciudadano. Vol. 18. Reverté. Barcelona.

Hernández, A., Alguacil, J., Medina, M. y Moreno, C. (2000). La ciudad de los ciudadanos. Ministerio de Fomento (1997), Madrid. Disponible en http://habitat.aq.upm.es/ aciudad/aciudad.pdf

Jacobs, J. M., Cairns, S., y Strebel, I. (2007). 'A tall story... but, a fact just the same': The Red Road high-rise as a black box. Urban Studies, 44(3), 609-629.

Kapstein, P. (2010). Vulnerabilidad y Periferia Interior. Cuadernos de Investigación Urbanística Instituto Juan de Herrera Madrid, Vol. 71.

Lerner, J. (2006). Charla "Cómo pensar una ciudad" (Buenos Aires). Consultado el 20 de octubre de 2014. Disponible en https://www.youtube.com/watch?v=lXGYoX-wdjI

Max-Neef, M. (1998). Desarrollo a escala humana (primera ed. 1986). Icaria, Barcelona. 
Mongil, D. (2010). Intervención integral en barrios: conceptos, instrumentos y elementos de mejora. Ciudades núm. 13, pp. 139-161, Universidad de Valladolid.

Morón, A. (2005). Pedagogía Social. Apuntes curso 2004-2005. Consultado el 15 de mayo de 2015. Disponible en http://es.slideshare.net/YAVAROS/pedagogia-social-12245179

Portas, N. (2003). El surgimiento del proyecto urbano. Perspectivas Urbanas/Urban Perspectives. núm. 3. Barcelona.

Ramírez, M.J. (2014). Acupuntura urbana. Estrategias de equilibrio territorial en Antofagasta. Seminario de Investigación en Arquitectura y Urbanismo, tutelado por Paula Kapstein, Universidad Católica del Norte (Chile), Diciembre de 2014. Documento no publicado.

Uribe, I., Molina, G. y Correa, M. (2011). Ética urbana. Editorial Universidad Pontificia Bolivariana, Medellín. 
Este artículo forma parte de:

\section{REVISTARQUIS}

Para más información, artículos, e instructivo de publicación, visite:

www.arquis.ucr.ac.cr/revistarquis.html 